\title{
SPATIAL CORRELATION FOR CORRELATED SCATTERERS
}

\author{
Terence Betlehem*
}

Research School of Information

Science and Engineering, Australian National University, Australia

terence.betlehem@anu.edu.au

\begin{abstract}
This paper investigates the correlations between sensor signals in multipath environments created by correlated scatterers. We derive a closed form expression for the correlation in fields created by arbitrary scatterer correlations and scatterer powers, using Fourier techniques, and propose reasonable function forms for scatterer correlation. Simulations show notable differences from the uncorrelated scatterer case.
\end{abstract}

\section{INTRODUCTION}

Multiple sensor systems have received much attention recently. An important characteristic of such systems in multipath environments is the level of correlation in the signals between sensors. This correlation dictates performance in MIMO systems and adaptive array processing algorithms. In this paper, we calculate correlation in general multipath environments.

For environments where multipaths propagate in uniformly from all directions, a half wavelength sensor spacing yields uncorrelated received signals [1]. Multipath is often more accurately modelled as coming from a subset of directions however. This causes more strongly correlated received signals and was recently studied by Teal et al. [2].

To calculate spatial correlation, [2] applied the wide-sense stationary uncorrelated scatterer (WSSUS) model [3], which assumes different scatterers (i.e. multipath gains) are uncorrelated. Direction-of-arrival and adaptive beamforming literature concedes this assumption is not strictly true, as coherent sources cause several subspace-based techniques to fail $[4,5]$.

In this paper, we derive a closed form expression for the spatial correlation between any two points in a wave field due to correlated scatterers. A small correlation between adjacent scatterers can cause significant deviations in spatial correlation from the WSSUS case. In the following analysis, we restrict attention to 2D, with obvious extension to 3D.

\footnotetext{
* This work was partially funded by Australian Research Council Discovery Grant number DP0343804.

†Thushara Abhayapala also has association with the Research School of Information Sciences and Engineering.
}

\author{
Thushara D. Abhayapala ${ }^{\dagger}$
}

\author{
Wireless Signal Processing Program, \\ National ICT Australia Ltd. \\ Canberra, Australia \\ thushara.abhayapala@anu.edu.au
}

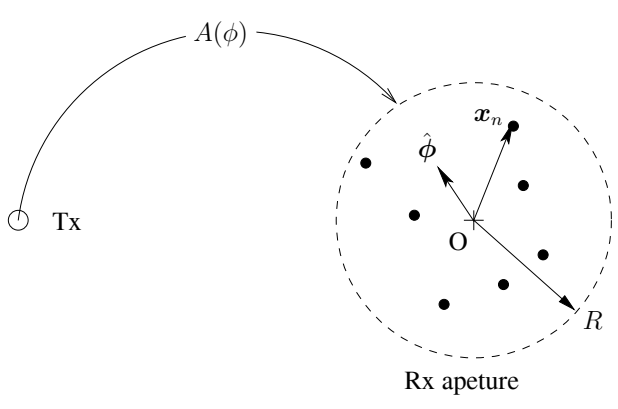

Fig. 1. Multipath channel model. Environmental scattering is modelled using transmitter-to-origin transfer function $A(\phi)$.

\section{CHANNEL MODEL}

Consider an antenna transmitting the unity amplitude narrowband signal $e^{i \omega t}$ over a slow fading channel. The signal propagates through a far-field scattering environment before reaching the receiver antennas. As shown in Fig. 1, each receiver is fixed at $\boldsymbol{x}_{n}$ within a circular aperture of radius $R$. The scattering environment is modelled with the scattering gain $A(\phi)$, defined as the transfer function between the transmitter and the receiver origin for a plane wave propagating in from direction $\hat{\phi}$ where in polar coordinates $\hat{\phi}=(1, \phi)$. The signal $s_{n}(t)$ captured by receiver antenna $n$ is written as:

$$
s_{n}(t)=e^{i \omega t} \int_{0}^{2 \pi} A(\phi) e^{-i k \boldsymbol{x}_{n} \cdot \hat{\boldsymbol{\phi}}} d \phi .
$$

For non-line-of-sight channels, the scattering gain $A(\phi)$ is assumed to be zero mean random variable. We are interested in the second order statistics of the scattering gain, which govern the properties of a wide variety of channels.

A useful quantifier of the channel properties is the normalized power density of scatterers

$$
\mathcal{P}(\phi) \triangleq \frac{E\left\{|A(\phi)|^{2}\right\}}{\int_{0}^{2 \pi} E\left\{|A(\phi)|^{2}\right\} d \phi} .
$$

This function, also known as the normalized power azimuth spectrum, indicates the relative power of the multipath coming from each angle $\phi$. 


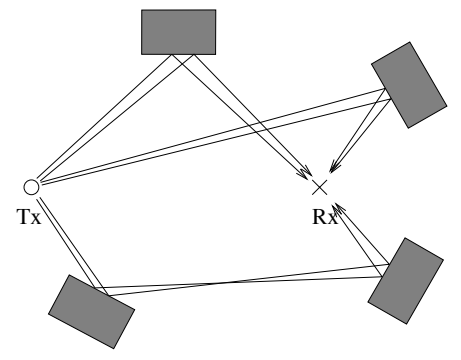

Fig. 2. Pairs of rays reflecting off homogeneous diffuse scatterers. At the receiver, rays have similar amplitudes and phases.

In this paper, we introduce the concept of scatterer correlation. Previously in the WSSUS model, the scatterers were assumed uncorrelated $E\left\{A(\phi) A^{*}(\varphi)\right\}=0$ for $\phi \neq \varphi$. We consider the general case of correlated scatterers, with correlation:

$$
\rho_{S}(\phi, \varphi) \triangleq \frac{E\left\{A(\phi) A^{*}(\varphi)\right\}}{\sqrt{E\left\{|A(\phi)|^{2}\right\} E\left\{|A(\varphi)|^{2}\right\}}} .
$$

We name the new model the correlated scatterer (CS) model ${ }^{1}$.

The intuition behind the CS model is justified thusly. When rays reflect non-specularly off scatterers, the rays can reach the receiver from two slightly different paths (Fig. 2). The path lengths are almost identical, and for homogenous scatterer, absorption losses are the same. Received amplitudes and phases at the receiver are almost identical. Statistically, these scatterers are correlated.

\section{FOURIER ANALYSIS OF MODEL PARAMETERS}

In this paper, we explore the spatial correlation in terms of the channel model parameters $\mathcal{P}(\phi)$ and $\rho_{S}(\phi, \varphi)$. We perform the Fourier expansions of these parameters.

\subsection{Root Power Density of Scatterers}

From the presence of $\sqrt{E\left\{|A(\phi)|^{2}\right\}}$ in (3) and the linear dependence of $E\left\{|A(\phi)|^{2}\right\}$ on power density of scatterers $\mathcal{P}(\phi)$, it is convenient to compute the Fourier expansion of root power density:

$$
\begin{gathered}
\sqrt{\mathcal{P}(\phi)}=\frac{1}{2 \pi} \sum_{m=-\infty}^{\infty} \gamma_{m} e^{i m \phi}, \\
\gamma_{m}=\int_{0}^{2 \pi} \sqrt{\mathcal{P}(\phi)} e^{-i m \phi} d \phi .
\end{gathered}
$$

Since root power density is real, $\gamma_{m}^{*}=\gamma_{-m}$.

\footnotetext{
${ }^{1}$ In the CS model, we can no longer apply the term wide-sense statioinary used in the WSSUS model. For a moving antenna array, $E\left\{s_{p}(t) s_{q}^{*}(t+\tau)\right\}$ is no longer invariant in $t$.
}

\subsection{Scatterer Correlation}

The scatterer correlation is a function of two angular variables. Hence we consider the double Fourier expansion

$$
\begin{gathered}
\rho_{S}(\phi, \varphi)=\frac{1}{(2 \pi)^{2}} \sum_{m=-\infty}^{\infty} \sum_{m^{\prime}=-\infty}^{\infty} \xi_{m m^{\prime}} e^{i\left(m \phi-m^{\prime} \varphi\right)}, \\
\xi_{m m^{\prime}}=\int_{0}^{2 \pi} \int_{0}^{2 \pi} \rho_{S}(\phi, \varphi) e^{-i\left(m \phi-m^{\prime} \varphi\right)} d \phi d \varphi .
\end{gathered}
$$

From (3), we observe the following properties of the angular correlation coefficient: (i) $\rho_{S}(\phi, \phi) \equiv 1$, (ii) $\rho_{S}(\phi, \varphi)=$ $\rho_{S}^{*}(\varphi, \phi)$ and (iii) $\left|\rho_{S}(\phi, \varphi)\right| \leq 1$. From these we can show the Fourier coefficients must satisfy $\sum_{m=-\infty}^{\infty} \xi_{m(m+n)}=$ $\delta_{n 0}$ for any integer $n$ and $\xi_{m\left(-m^{\prime}\right)}=\xi_{m^{\prime}(-m)}$ where $\delta_{n n^{\prime}}$ is the Kronecker delta function.

\section{SPATIAL CORRELATION}

Spatial correlation is defined as the correlation coefficient between two received signals captured by omnidirectional sensors in the wave field:

$$
\rho\left(\boldsymbol{x}_{p}, \boldsymbol{x}_{q}\right) \triangleq \frac{E\left\{s_{p}(t) s_{q}^{*}(t)\right\}}{\sqrt{E\left\{\left|s_{p}(t)\right|^{2}\right\} E\left\{\left|s_{q}(t)\right|^{2}\right\}}} .
$$

We derive an expression for the covariance between receiver signals $E\left\{s_{p}(t) s_{q}^{*}(t)\right\}$. The expression for spatial correlation can then be obtained by substituting this back into (6).

\section{Theorem (Signal Covariance for CS Channels)}

The correlation between received signals $s_{p}(t)$ and $s_{q}(t)$ is given by:

$$
E\left\{s_{p}(t) s_{q}^{*}(t)\right\}=\mathcal{A} \sum_{n=-\infty}^{\infty} \sum_{n^{\prime}=-\infty}^{\infty} \zeta_{n n^{\prime}} \mathcal{J}_{n}\left(\boldsymbol{x}_{p}\right) \mathcal{J}_{n^{\prime}}^{*}\left(\boldsymbol{x}_{q}\right)
$$

where

$$
\begin{aligned}
\zeta_{n n^{\prime}}= & \sum_{m=-\infty}^{\infty} \sum_{m^{\prime}=-\infty}^{\infty} \xi_{m m^{\prime}} \gamma_{m+n}^{*} \gamma_{m^{\prime}+n^{\prime}} \\
& \mathcal{J}_{n}(\boldsymbol{x}) \triangleq i^{n} J_{n}(k x) e^{i n \theta_{x}}
\end{aligned}
$$

where in polar coordinates $\boldsymbol{x}=\left(x, \theta_{x}\right)$,

$$
\mathcal{A} \triangleq \int_{0}^{2 \pi} E\left\{|A(\phi)|^{2}\right\} d \phi
$$

is a constant normalizing term, and $J_{n}(x)$ is the Bessel function of the first kind of order $n . \gamma_{m}$ and $\xi_{m m^{\prime}}$ are defined in (4) and (5) respectively.

The proof for this theorem is in the appendix. The resulting spatial correlation expression is more complicated 


\begin{tabular}{|l|c|c|}
\hline Distribution & Angular Power $\mathcal{P}(\phi)$ & Root Power Coef. $\gamma_{m}$ \\
\hline Isotropic & $\begin{cases}\frac{1}{2 \Delta}, \quad\left|\phi-\varphi_{0}\right| \leq \Delta, & \sqrt{2 \pi} \delta_{m 0} \\
0, & \text { otherwise. }\end{cases}$ & $\sqrt{2 \Delta} e^{-i m \varphi_{0}} \operatorname{sinc}(m \Delta)$ \\
Uniform limited & $\begin{cases}\frac{1}{2 \pi I_{0}(\kappa)} e^{\kappa \cos \left(\phi-\varphi_{0}\right)}, & \left|\phi-\varphi_{0}\right| \leq \pi, \\
0, & \text { otherwise. }\end{cases}$ & $\sqrt{\frac{2 \pi}{I_{0}(\kappa)}} e^{-i m \varphi_{0}} I_{-m}\left(\frac{\kappa}{2}\right)$ \\
Lon-Mises & $\begin{cases}\frac{Q_{1}}{\sqrt{2} \sigma} e^{-\sqrt{2}\left|\phi-\varphi_{0}\right| / \sigma}, & \left|\phi-\varphi_{0}\right| \leq \frac{\pi}{2}, \\
0, & \text { otherwise. }\end{cases}$ & $Q_{2} e^{-i m \varphi_{0}} \frac{1-(-1)^{\left\lceil\frac{m}{2}\right\rceil} \eta F_{m}}{\left(1+2 \sigma^{2} m^{2}\right)(1-\eta)}$ \\
\hline
\end{tabular}

Table 1. Popular angular power distributions and associated coefficients of root power. $I_{m}(\cdot)$ is the modified Bessel function of the first kind. For the Laplacian distribution, $\eta=e^{-\pi /(2 \sqrt{2} \sigma)}, F_{m}=1$ for $m$ even, $F_{m}=\sqrt{2} m \sigma$ for $m$ odd and $Q_{1}=$ $(1-\exp (-\pi / \sqrt{2} \sigma))^{-1}$ and $Q_{2}=2^{5 / 4} \sqrt{\sigma / Q_{1}}$ are normalization constants.

than the WSSUS case [2], since the received signal energy $E\left\{\left|s_{p}(t)\right|^{2}\right\}$ is now a function of position.

Due to the bandpass property of Bessel functions, we can truncate each summation in (7) to a small number $N=$ $\lceil e k R / 2\rceil$ of terms, without loss of accuracy [6]. The theorem is hence elegant, as the covariances are governed by $(2 N+1)^{2} \zeta_{n n^{\prime}}$ coefficients. Once computed, the covariance between any two sensor signals is readily determined.

In the WSSUS case $\rho_{S}(\phi, \varphi)=\delta_{\phi \varphi}$ and (8) reduces to $\zeta_{n n^{\prime}}=\sum_{m=-\infty}^{\infty} \gamma_{m+n}^{*} \gamma_{m+n^{\prime}}$ for which $\zeta_{n n^{\prime}}=\zeta_{\left(n-n^{\prime}\right) 0}$. One can show $\zeta_{n 0}$ is the Fourier coefficient of $\mathcal{P}(\phi)$ and that the theorem is consistent with the WSSUS result in [2].

\section{FUNCTIONAL FORMS OF MODEL PARAMETERS}

Angular power distributions have been well discussed in the literature $[1,7,8,9]$. Angular power is usually parametrised with the mean direction $\varphi_{0}$ and the angular spread $\sigma$, defined as the root variance of power density. Common examples are summarized in Table 1, along with corresponding $\gamma_{m}$ coefficients. Coefficients were derived following [2].

The functional form of scatterer correlation is comparatively unstudied. Intuitively, if scattered rays close in angle have arrived from the same scattering object, they will be strongly correlated in magnitude and phase. We propose a $\rho_{S}(\phi, \varphi)$ that is close to 1 for small $|\phi-\varphi|$ and then smoothly decreases to 0 .

For scatter correlation only dependent on the angular difference $\phi-\varphi, \rho_{S}(\phi, \varphi)=\rho_{S}(\phi-\varphi)$, the Fourier coefficients reduce to $\xi_{m m^{\prime}}=\xi_{m} \delta_{m m^{\prime}}$ where $\xi_{m} \triangleq \xi_{m m}$. For simplicity, we explore angular correlation given by the Gaussian density function:

$$
\rho_{S}(\phi, \varphi)=e^{-(\phi-\varphi)^{2} / 2 \sigma_{S}^{2}}
$$

where $\sigma_{S}$ is the standard deviation of the angular correlation. Within a scaling constant, $\xi_{m}=e^{-m^{2} \sigma_{S}^{2} / 2}$. More general angular correlation will be considered in future work.

\section{EXAMPLES}

In Fig. 3(a), we plot the spatial correlation at different points in the field for a sensor pair with constant separation $2 \lambda$ using the Gaussian scatterer correlation (11) with $\sigma_{S}=2^{\circ}$. WSSUS spatial correlation only depends upon the displacement between sensors. With CS, we see variation of the spatial correlation with sensor pair position, for only a small $\sigma_{S}$.

Fig. 3(b) and (c) plots spatial correlation for uniformly distributed scatterers possessing (b) Gaussian angular correlation and (c) the randomly-chosen angular correlation shown in Fig. 3(d). Whilst Gaussian correlation yields modest differences from the WSSUS case, the random angular correlation causes significant divergence. In Fig. 3(c) the zeros in the isotropic $(\Delta=2 \pi)$ case no longer corresponds to those in Jakes model. In fields generated from isotropically distributed correlated scatterers, half wavelength spacings do not always yield uncorrelated sensor signals.

\section{CONCLUSION}

A closed form expression for spatial correlation has been derived for the case of correlated scatterers, using Fourier techniques. Simulations show that (i) for small angular correlation in scatterers, the spatial correlation can be significantly different from the uncorrelated scatterer case, and (ii) half wavelength sensor spacings do not always yield uncorrelated signals in fields from isotropic scatterer power.

\section{APPENDIX}

Proof

We calculate the expectation $E\left\{s_{p}(t) s_{q}^{*}(t)\right\}$ directly from (1):

$$
\begin{aligned}
E\left\{s_{p}(t) s_{q}^{*}(t)\right\}= & \int_{0}^{2 \pi} \int_{0}^{2 \pi} E\left\{A(\phi) A^{*}(\varphi)\right\} \times \\
& e^{-i k \boldsymbol{x}_{p} \cdot \hat{\boldsymbol{\varphi}}} e^{i k \boldsymbol{x}_{q} \cdot \hat{\boldsymbol{\phi}}} d \phi d \varphi .
\end{aligned}
$$

First rearranging (2), we see that

$$
E\left\{|A(\phi)|^{2}\right\}=\mathcal{A P}(\phi),
$$




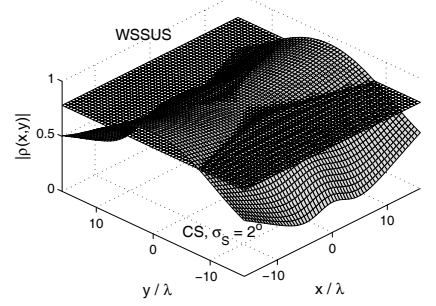

(a)

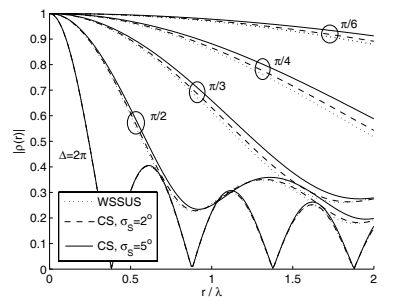

(b)

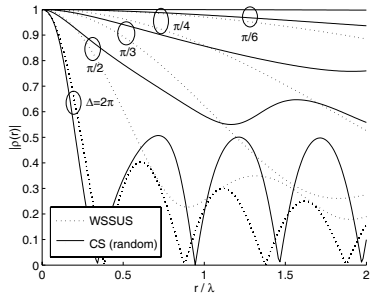

(c)

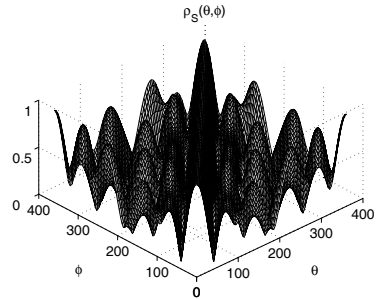

(d)

Fig. 3. Spatial correlation for a sensor pair as (a) the pair is shifted through a field created from Von-Mises distributed scatterers ( $\kappa=5, \varphi_{0}=\pi / 2$ ) with $2 \lambda$ sensor separation, (b) and (c) a sensor is fixed at the origin while the second sensor moved along the y-axis with uniformly distributed scatterers (half-width $\Delta, \varphi_{0}=\pi / 2$ ) and sensor separation $r$ using (b) Gaussian and (c) a random angular correlation. (d) The random angular correlation function $\rho_{S}(\theta, \phi)$ used to generate the field in (c).

where $\mathcal{A}$ is defined in (10). Similarly rearranging (3) and inserting (13),

$$
E\left\{A(\phi) A^{*}(\varphi)\right\}=\mathcal{A} \sqrt{\mathcal{P}(\phi)} \sqrt{\mathcal{P}(\varphi)} \rho_{S}(\phi, \varphi) .
$$

Then inserting (14) into (12), followed by the Fourier expansions of $\rho_{S}$ and $\mathcal{P}$ and the Jacobi-Anger expression [10],

$$
e^{i k \boldsymbol{x} \cdot \hat{\boldsymbol{\phi}}}=\sum_{n=-\infty}^{\infty} i^{n} J_{n}(k x) e^{i n\left(\theta_{x}-\phi\right)},
$$

where $\boldsymbol{x} \triangleq\left(x, \theta_{x}\right)$, into (12) and rearranging:

$$
\begin{aligned}
& E\left\{s_{p}(t) s_{q}^{*}(t)\right\}=\mathcal{A} \sum_{\ell} \gamma_{\ell} \sum_{\ell^{\prime}} \gamma_{\ell^{\prime}} \sum_{m} \sum_{m^{\prime}} \xi_{m m^{\prime}} \sum_{n} \sum_{n^{\prime}} \\
& \quad \times \frac{1}{(2 \pi)^{2}} \int_{0}^{2 \pi} \int_{0}^{2 \pi} e^{i\left(\ell \phi+\ell^{\prime} \varphi\right)} e^{i\left(m \phi-m^{\prime} \varphi\right)} e^{i\left(n-n^{\prime}\right) \phi} d \phi d \varphi \\
& \times \mathcal{J}_{n}^{*}\left(\boldsymbol{x}_{p}\right) \mathcal{J}_{n^{\prime}}\left(\boldsymbol{x}_{q}\right),
\end{aligned}
$$

where $\mathcal{J}_{n}(\boldsymbol{x})$ is defined in (9). The integrals are only nonzero when $\ell+m+n=0$ and $\ell^{\prime}-m^{\prime}-n^{\prime}=0$ in which case each integral evaluates to $2 \pi$. Hence:

$$
\begin{aligned}
E\left\{s_{p}(t) s_{q}^{*}(t)\right\}= & \mathcal{A} \sum_{n} \sum_{n^{\prime}} \mathcal{J}_{n}^{*}\left(\boldsymbol{x}_{p}\right) \mathcal{J}_{n^{\prime}}\left(\boldsymbol{x}_{q}\right) \\
& \times \underbrace{\sum_{m} \sum_{m^{\prime}} \xi_{m m^{\prime}} \gamma_{-m-n} \gamma_{m^{\prime}+n^{\prime}}}_{\zeta_{n n^{\prime}}} .
\end{aligned}
$$

The result in (8) follows after applying property $\gamma_{-\ell}=\gamma_{\ell}^{*}$.

\section{REFERENCES}

[1] W. C. Jakes, "Microwave mobile communications," Wiley, New York, 1974.

[2] P. Teal and T. A. Abhayapala, "Spatial correlation in non-isotropic scattering scenarios," in Proc. IEEE International Conference on Acoustics, Speech and Signal Processing, 2002, vol. III, pp. 2833 - 2866.
[3] P. A. Bello, "Characterization of randomly time-variant linear channels," IEEE Trans. Comm. Sys., vol. 11, pp. $360-393,1963$.

[4] T. J. Shan and T. Kailath, "Adaptive beamforming for coherent signals and interference," IEEE Trans. on $A S S P$, vol. 33, no. 3, pp. $527-536,1985$.

[5] V. U. Reddy, A. Paulraj, and T. Kailath, "Performance analysis of the optimum beamformer in the presence of correlated sources and its behaviour under spatial smoothing," IEEE Trans. on ASSP, vol. 35, no. 7, pp. 927 - 937, 1987.

[6] H. M. Jones, R. A. Kennedy, and T. D. Abhayapala, "On dimensionality of multipath fields: spatial extent and richness," in Proc. IEEE International Conference on Acoustics, Speech and Signal Processing, Orlando, 2002, vol. III, pp. 2837 - 2840.

[7] J. Saltz and J. H. Winters, "Effect of fading correlation on adaptive arrays in digital mobile radio," IEEE Trans. Veh. Technol., vol. 42, pp. 1049 - 1057, 1994.

[8] A. Abdi, J. A. Barger, and M. Kaveh, "A parametric model for the distribution of the angle of arrival and the associated correlation and power spectrum at the mobile station," IEEE Trans. Veh. Technol., vol. 51, no. 3, pp. 425 - 434, 2002.

[9] K. L. Pederson, P. E. Morgensen, and B. H. Fleury, "Power azimuth spectrum in outdoor environments," IEEE Electron. Lett., vol. 33, no. 18, pp. 1583 - 1584, 1997.

[10] D. Colton and R. Kress, Inverse acoustic and electromagnetic scattering theory, Springer-Verlag, Berlin, 1992. 